\title{
Analytical Consideration of Surface Dose and Kerma for Megavoltage Photon Beams in Clinical Radiation Therapy
}

\author{
Mohammad Javad Tahmasebi Birgani ${ }^{1,2}$, Mohammad Ali Behrooz ${ }^{1}$, Sasan \\ Razmjoo $^{2}$, Mansour Zabihzadeh ${ }^{1}$, Jafar Fatahiasl ${ }^{1,2}$, Reza Maskni ${ }^{1}$, Neda \\ Abdalvand $^{1,2 *}$, Zeynab Asgarian ${ }^{1}$, Azin Shamsi ${ }^{1,2}$
}

\begin{abstract}
Background: In radiation therapy, estimation of surface doses is clinically important. This study aimed to obtain an analytical relationship to determine the skin surface dose, kerma and the depth of maximum dose, with energies of 6 and 18 megavoltage (MV). Materials and Methods: To obtain the dose on the surface of skin, using the relationship between dose and kerma and solving differential equations governing the two quantities, a general relationship of dose changes relative to the depth was obtained. By dosimetry all the standard square fields of $5 \mathrm{~cm} \times 5 \mathrm{~cm}$ to $40 \mathrm{~cm} \times 40 \mathrm{~cm}$, an equation similar to response to differential equations of the dose and kerma were fitted on the measurements for any field size and energy. Applying two conditions: a) equality of the area under dose distribution and kerma changes in versus depth in 6 and $18 \mathrm{MV}$, b) equality of the kerma and dose at $x=d_{\text {max }}$ and using these results, coefficients of the obtained analytical relationship were determined. By putting the depth of zero in the relation, amount of PDD and kerma on the surface of the skin, could be obtained. Results: Using the MATLAB software, an exponential binomial function with R-Square $>0.9953$ was determined for any field size and depth in two energy modes 6 and 18MV, the surface PDD and kerma was obtained and both of them increase due to the increase of the field, but they reduce due to increased energy and from the obtained relation, depth of maximum dose can be determined. Conclusions: Using this analytical formula, one can find the skin surface dose, kerma and thickness of the buildup region.
\end{abstract}

Keywords: Dosimetry - skin surface dose - kerma - percentage depth dose - buildup region

Asian Pac J Cancer Prev, 17 (1), 153-157

\section{Introduction}

High-energy photons generated by a medical linear accelerators are used in radiation therapy. With an increasing energy of these radiations, the penetration power of photons and secondary electrons will be increased and as a result the point of maximum dose (d_max) is placed in more depth (Apipunyasopon et al., 2013).Depending on the amount of energy, dose build up region occurs for $\mathrm{x}$-rays megavoltage in the first depths of the entrance surface of phantom or skin. Dose build up region are a causes the skin sparing effect in megavoltage beams and makes them suitable for delivering the lethal dose to deep tumors as well as reaching the lowest dose in the surface layers of the skin (Butson et al., 1997; Ravikumar and Ravichandran, 2000). On the other hand, low-energy photons and electron contamination causes an increase in the dose of skin, and loss or decrease in the skin sparing effect in the treatment with megavoltage beams (Ravikumar and Ravichandran, 2000).

Accumulated dose at the boundary between the air and the patient's skin, which is known as the surface dose, is not clinically negligible and can be a limiting factor in dose delivery to deep tumors. As a result, determining the exact amount of surface dose for assessment of skin damage is essential in the planning of a proper radiation therapy technique (Devic et al., 2006). On the other hand, dosimetry of buildup region is important to determine the bolus thickness needed to increase the surface dose (Klein et al., 2003).

From a physical point of view, the surface dose is the energy deposited within a very small mass of tissue at the surface of phantom, but there is no dosimeter with such a sensitive volume and surface dose measured by the dosimeters indicates dose measured at the their effective point of measure (Devic et al., 2006). In clinical conditions, the curves of PDD are obtained in a water phantom mainly using a computerized scanning system. But due to the high uncertainty of dose calculation in the first few millimeters beneath the surface of the water using this method, high-precision measurement cannot be obtained (Ishmael Parsai et al.,2008). A variety of dosimeters, such

${ }^{1}$ Deprtment of Medical Physisc, School of Medicine, ${ }^{2}$ Department of clinical Oncology, Golestan Hospital, Ahavaz Jundishapur University of Medical sciences, Ahvaz, Iran*For correspondence: abdalvandneda@yahoo.com 
as the radiochromic films (Bilge et al., 2009), parallel plates chambers (Tannous et al., 1981), TLD (Charles and Khan, 1978; Lin et al., 2001), diodes (Jornet et al., 2000) and MOSFET (Scalchi et al., 2005) can be used. Surface dose measurements are often compared with extrapolation chambers (Nilsson and Montelius, 1986; Cora and Francescon, 1995). These measurements are time-consuming because these chambers by extrapolating different points, measure dose several times to obtain the skin surface dose. While extrapolation chambers are appropriate in experimental conditions, but in the clinical applications that we require skin or surface dose for each patient, they would be impractical (Devic et al., 2006). So we need an analytical relationship between dose and kerma to get the surface dose so that errors caused by the use of dosimeters are avoided.

The absorbed dose that is depended on photon fluence of the total volume under radiation, and not merely the photon fluence at the point, is composed of two primary and secondary components. The initial dose, is dose deposited by electrons generated by photons in each the point that already have not had the in teraction in volume under radiation and the secondary dose comes from the interaction of electrons by photons that already have had at least one interaction (Loevinger, 1981).

Kerma is the sum of initial kinetic energies of electrons released per unit mass by photon beams and because its radiant part escapes from the absorbed dose region, the absorbed dose is related to the collision part of kerma and in this regard, three states arise:

Near the entrance surface, where the photon beam diameter appreciably is less than the maximum electron range in the medium and the electron equilibrium does not happen, the ratio of the dose to the collision part of kerma $(\beta)$ will be less than 1 . At a depth of greater than the maximum electron range and assuming that the photon attenuation is negligible in the desired area, there will be an equilibrium of charged particles and $\beta$ would equal to one; finally, at a depth greater than the maximum electron range, which is called transient electronic equilibrium, due to the combined effect of the photon beams attenuation and mainly forward moving of secondary electrons, $\beta$ will be greater than one (Loevinger, 1981).

From a mathematical point of view, kerma and absorbed dose curves are similar to transient equilibrium in radioactive nuclei, and here the kerma curve is similar to the parent activity that decays with the time, and dose curve is similar to the daughter's activity, the difference is that unlike the daughter's activity that is initially zero, The amount of surface dose always greater than zero due to the incident and/or back scattered charged particles(Attix, 2008).

The aim of this study was to obtain an analytical relationship to measure the dose and percentage depth dose (PDD) for all fields and all depths, including zero depth (surface of the skin) with regard to the differential equations that govern the quantities of dose and kerma and parameterize the relationship using standards radiation fields (the square fields) at all therapeutic depths and 6 and 18 MVphoton energies of Siemens Primus Plus linear accelerator in Golestan Hospital, Ahvaz, Iran. This relationship can contain information about the distribution of dose throughout the treatment volume that one of them is the skin surface dose parameter.

\section{Materials and Methods}

\section{A. Theory}

Due to the collision of megavoltage photons with equivalent body phantom, the electrons from surface layer of phantom are emitted by absorbing the radiation photon energy that this causes ionization in the phantom; therefore, the phantom surface absorbs the amount of photon energy and a number from substrates return to the back and deposit their energy at the surface (surface dose). The calculation of the dose can be performed with the help of physical analysis and mathematics of the interaction between photons with the phantoms. Free electrons from the surface layer of phantom with roughly equivalent energy to photons become a factor of new interactions in the next layer of the phantom. The energy available for electrons (kerma) becomes a factor for a new absorbed dose in phantom and so the process can continue. Thus, the amount of kerma constantly decrease and the amount of absorbed dose is added until the electronic equilibrium are met, namely almost the amount of kerma and the dose becomes almost equal $(\beta=1)$. The law governing these interactions is exponential attenuation law and one can write as the following;

$$
\frac{\mathrm{dK}}{\mathrm{d} \chi}=-\mu_{\mathrm{k}} \mathrm{K} \rightarrow \mathrm{K}(\chi)=\mathrm{K}(0) \mathrm{e}^{-\mu \mathrm{k} \chi},
$$

where $K$ is the kinetic energy of the electrons and $\mu_{\mathrm{k}}$ is the attenuation coefficient of this energy.On the other hand, the law governing the dose or the absorbed photon energy and convertit to dose, according to the exponential attenuation law, is as follows:

$$
\frac{\mathrm{dK}}{\mathrm{d} \chi}=-\mu_{\mathrm{p}} \mathrm{D}+\gamma \mathrm{K}(\chi)
$$

where $\mathrm{D}$ is absorbed dose and $\mu_{\mathrm{p}}$ is its attenuation coefficient and $\gamma$ is the rate of convert it the kerma toabsorbeddose. Replacing thevalue of $\mathrm{K}(\mathrm{x})$ in theEq. (2), it gives :

$$
\frac{\mathrm{dK}}{\mathrm{d} \chi}+\mu_{\mathrm{p}} \mathrm{D}=\gamma \mathrm{K}_{(0)} \mathrm{e}_{\mathrm{k}}^{-\mu^{\alpha}},
$$

This is a first-order linear differential equation as the form of $\frac{\mathrm{dK}}{\mathrm{d} x}+\mathrm{R}_{(\mathrm{x})} \mathrm{y}=\mathrm{Q}_{(\mathrm{x})}$ and the answer to this equation is as the following:

$$
y_{(x)}=e^{-\int R d x}\left[C+\int Q_{(x)} e^{\int R d x} d x\right]
$$

where $\mathrm{C}$ is an integral constant and byusing physical condition is determined. Then the answer of Eq. (3) becomes:

$$
\mathrm{D}_{(\mathrm{x})}=\mathrm{Ce}^{-\mu_{\mathrm{p}}} \chi-\frac{\gamma \mathrm{K}(0)}{\mu_{\mathrm{k}}-\mu_{\mathrm{p}}} \mathrm{e}^{-\mu_{\mathrm{k}} \chi}
$$

Because at the $\mathrm{d}_{\max }$ depth, dose should be maximum, so 


$$
\left.\frac{\mathrm{dK}}{\mathrm{d} \chi}\right|_{\chi \mathrm{x}=\mathrm{d}_{\max }} ^{@}=0 .
$$

By applying the condition (5) the constant of $\mathrm{C}$ is determined and with replacing of it Eq.(4) for each field size 1 one can find:

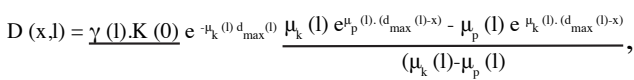

$$
\begin{aligned}
& K(x, 1)=K(0,1) e^{-\mu_{k}(1) x} . \quad(6 . b)
\end{aligned}
$$

By putting $x=d_{\text {max }}$ in the Eq.(6.a), D $\left(x=d_{\max }\right)$ is obtained. So:

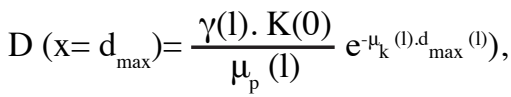

$$
\begin{aligned}
& \text { and because of PDD }(x, 1)=\frac{\mathrm{D}(\mathrm{x}, \mathrm{l})}{\mathrm{D}^{\left(\mathrm{x}=\mathrm{d}_{\max }\right)}} \text { : }
\end{aligned}
$$

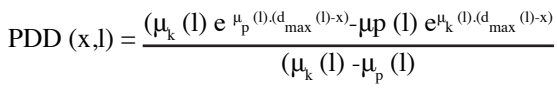

\section{B. Dosimetry and measurement}

In this research, we used the Omni-accept pro 6.5 software, $0.13 \mathrm{cc}$ Scanditronix ioniziation chamber and Blue phantom $\left(50 \times 50 \times 50 \mathrm{~cm}^{3}\right)$. In all standard fields from $5 \times 5 \mathrm{~cm}^{2}$ to $40 \times 40 \mathrm{~cm}^{2}$ and to a depth of $25 \mathrm{~cm}$, the PDD value was measured as follows.

A dosimeter as a monitor was installed on the moveable phantom arm and an other dosimeter as a reference dosimeter was installed on the linear accelerator. Siemens Primus Plus. The MCU500E machine was used as a computer interface to control the movements of aphantomarmandreading of the output dosimeter readings. The ratio of the readings of two dosimeter was recorded by the electrometer at any point and then the amoun of frecorded in any depth was divided into the value recorded at $\mathrm{x}=\mathrm{d}_{\max }$ in each field that PDD in any depth was obtained.

With the help of software of MATLAB 2010a, the best fitting on the PDD data in each field according to the depth is achieved. The results of the fitting will be equivalent to Eq. (8) that from this equivalence, values of its coefficients were determined.

Using the assumption that the amount of kerma and absorbed dose at a $d_{\text {max }}$ depth will be equal $(\beta=1)$, the $\gamma$ amount will be obtained as follows:

$$
\mathrm{K}\left(\mathrm{x}=\mathrm{d}_{\max }\right)=\mathrm{D}\left(\mathrm{x}=\mathrm{d}_{\max }\right) \rightarrow \gamma=\mu_{\mathrm{p}} .
$$

Due to the fact that the area under the kerma and dose changes curve should be the same, one can define a spatial displacement coefficient(u) to correct the experimental data compliance;

$$
\mathrm{S}_{\mathrm{K}}=\mathrm{S}_{\mathrm{D}} \rightarrow \int \mathrm{K} \cdot \mathrm{dx}=\int \mathrm{D} \cdot \mathrm{dx} \rightarrow \mathrm{u}(\mathrm{l})=\mathrm{d}_{\text {max }}(1)-\frac{\ln \mu_{\mathrm{k}}-\ln \mu^{\mathrm{p}}}{\mu_{\mathrm{k}}-\mu_{\mathrm{p}}}
$$

Therefore, constant values achieved from a measurement, must be shiftted with a namount of $u_{(1)}$. The value of $\mathrm{K}_{(0)}$ that for each field is dependent on the amount of the prescribed dose at the desired depth of $\mathrm{x}$ is determined by using Eq. (6.a) as the following:

$$
\mathrm{K}(0)=\mathrm{K}(0)=\frac{100}{\operatorname{PDD}(\mathrm{x})} \mathrm{e}_{\mu} \mathrm{K} \cdot \mathrm{d}_{\max } .
$$

Then using $\mu_{\mathrm{k}}$ and $\mathrm{K}_{(0)}$, at any desired depth for each field, kerma value can be determined.

\section{Results}

As a result of fitting of PDD of each field with MATLAB software, a binomial exponential function with R-square $>0.9953$ for energies of 6 and $18 \mathrm{MV}$ will be in the form of PDD $(\mathrm{x})=\mathrm{Ae}^{\wedge}(-\mathrm{bx})-\mathrm{Ce}^{\wedge}(-\mathrm{dx})$ that it is well-matched the analytical Eq. (8). In each of the coefficients of A, b, C, d and d for the energies of 6 and $18 \mathrm{MV}$ separately, depending on the field size, we matched a polynomial function of $4^{\text {th }}$ degree, with R-square $>0.9907$ and by applying the displacement of $u$, the results will be as follows:

$$
\begin{aligned}
& \mathrm{A}(1)=\frac{\left(\mu_{\mathrm{k}}(1) \cdot \mathrm{e}^{\mu_{\mathrm{k}}(1) \cdot \mathrm{d}_{\max }(1)}\right.}{\left(\mu_{\mathrm{k}}(1)-\mu_{\mathrm{p}}(1)\right.} \\
& \mathrm{b}(\mathrm{l})=\mu_{\mathrm{p}}(1) \quad(12 \cdot \mathrm{b}) \\
& \mathrm{C}(\mathrm{l})=\frac{\left(\mu_{\mathrm{p}}(1) \cdot \mathrm{e}^{\mu_{\mathrm{k}}(1) \cdot \mathrm{d}_{\max }(1)}\right.}{\left.\mu_{\mathrm{k}}(1)-\mu_{\mathrm{p}}(1)\right)} \\
& \mathrm{d}(1)=\mu_{\mathrm{k}}(1)
\end{aligned}
$$

for $6 \mathrm{MV}(13 . \mathrm{a})$

$\rightarrow\left\{\begin{array}{l}\left(\mathrm{A}(1)=\left(-9.646 \times 10^{-6}\right) 1^{4}+\left(9.464 \times 10^{-4}\right) 1^{3}-\left(2.932 \times 10^{-2}\right) 1^{2}+\left(2.172 \times 10^{-1}\right) 1+114.3\right. \\ \mathrm{b}(1)=\left(5.841 \times 10^{-9}\right) 1^{4}-\left(8.676 \times 10^{-7}\right) 1^{3}+\left(5.319 \times 10^{-5}\right) 1^{2}-\left(1.67 \times 10^{-3}\right) 1+6.591 \times 10^{-2} \\ \left.\mathrm{C}(1)=\left(2.202 \times 10^{-5}\right) 1^{4}-\left(2.068 \times 10^{-3}\right)\right)^{3}+\left(6.345 \times 10^{-2}\right) 1^{2}-\left(5.574 \times 10^{-1}\right) 1+82.99 \\ \mathrm{~d}(1)=\left(9.103 \times 10^{-7}\right) 1^{4}-\left(8.408 \times 10^{-5}\right) 1^{3}+\left(2.599 \times 10^{-3}\right) 1^{2}-\left(2.593 \times 10^{-2}\right) 1+1.669\end{array}\right.$

for $18 \mathrm{MV}$ (13.b)

$\mathrm{A}(\mathrm{l})=\left(-3.282 \times 10^{-5}\right) 1^{4}+\left(2.948 \times 10^{-3}\right) 1^{3}-\left(8.09 \times 10^{-2}\right) 1^{2}+\left(4.843 \times 10^{-1}\right) 1+119.6$ $\rightarrow \quad \mathrm{b}(\mathrm{l})=\left(4.753 \times 10^{-9}\right) 1^{4}-\left(6.767 \times 10^{-7}\right) 1^{3}+\left(3.81 \times 10^{-5}\right) 1^{2}-\left(1.07 \times 10^{-3}\right) 1+4.943 \times 10^{-2}$ $\left\{(1)=\left(4.902 \times 10^{-5}\right) 1^{4}-\left(4.395 \times 10^{-3}\right) 1^{3}+\left(1.178 \times 10^{-1}\right) 1^{2}-5.689 \times 10^{-1}\right) 1-100.8$ $\mathrm{d}(\mathrm{l})=\left(1.226 \times 10^{-6}\right) 1^{4}-\left(1.113 \times 10^{-4}\right) 1^{3}+\left(3.003 \times 10^{-3}\right) 1^{2}-\left(1.409 \times 10^{-2}\right) 1+9.202 \times 10^{-1}$

By replacementthe coefficients obtainedfrom Eq.(13) and putting $x($ Depth $)=0$ inEq.(8), the amount ofPDDin the surface of the skin for each field lcan be obtainedas follows;

$$
\operatorname{PDD}(0,1)=\mathrm{A}(1)-\mathrm{C}(1)
$$

Its results for energies of 6 and $18 \mathrm{MV}$ are shown as in Table1.

Table 1. Percentage of surface depth dose based on the field size in energies of 6 and 18 MV Siemens primus Plus in Golestan Hospital, Ahvaz

\begin{tabular}{clc}
\hline Energy(MV) & & Side of Fields $(\mathrm{cm})$ \\
\hline 6 & 18 & \\
30.35 & 19.15 & 5 \\
30.34 & 20.36 & 10 \\
30.75 & 21.79 & 15 \\
31.19 & 23.45 & 20 \\
31.46 & 23.45 & 25 \\
31.57 & 23.5 & 30 \\
31.71 & 23.3 & 35 \\
32.21 & 23.28 & 40 \\
\hline
\end{tabular}


Table 2. The amont of kerma on the surface(cGy) with the prescribed dose of 100cGy to a depth of $10 \mathrm{~cm}$ based on field size in 6 and 18 MV energies of Siemens primus plus in Golestan Hospital, Ahvaz

\begin{tabular}{lcc}
\hline \multicolumn{2}{c}{ Energy(MV) } & Side of Fields $(\mathrm{cm})$ \\
\hline \multicolumn{1}{c}{6} & 18 & 5 \\
3508.44 & 2574.34 & 10 \\
3632.23 & 2830.23 & 15 \\
3799.865 & 3136.35 & 20 \\
3956.86 & 3389.96 & 25 \\
4073.03 & 3536.15 & 30 \\
4145.52 & 3574.67 & 35 \\
4197.75 & 3559.05 & 40 \\
4275.83 & 3595.47 & \\
\hline
\end{tabular}

Table 3. Depth of maximum dose $(\mathbf{c m})$ in size of field in energies of 6 and $18 \mathrm{MV}$ Siemens primus plus in Golestan Hospital,Ahvaz

\begin{tabular}{llc}
\hline \multicolumn{2}{c}{ Energy(MV) } & Side of Fields $(\mathrm{cm})$ \\
\hline 6 & 18 & \\
1.95 & 3.27 & 5 \\
2 & 3.159 & 10 \\
2.006 & 2.99 & 15 \\
1.997 & 2.86 & 20 \\
1.993 & 2.804 & 25 \\
1.997 & 2.809 & 30 \\
1.998 & 2.846 & 35 \\
1.973 & 2.847 & 40 \\
\hline
\end{tabular}

At a dose of $100 \mathrm{cGy}$ prescribed to a depth of $10 \mathrm{~cm}$, the kerma amount in the skin was obtained according to Eq. (11) the results are shown in Table 2.

Derivative of Eq. (8) compared to the depth, $\mathrm{d}_{\max }$ is obtained as follows:

$$
\frac{\partial \mathrm{PDD}}{\partial \mathrm{x}}=0 \rightarrow \mathrm{d}_{\text {max }}(\mathrm{l})=\frac{\ln \left(\mathrm{A}_{1} \cdot \mathrm{b}_{1}\right)-\ln \left(\mathrm{C}_{1} \cdot \mathrm{d}_{1}\right)}{\mathrm{b}_{1}-\mathrm{d}_{1}}
$$

And with substituting the coefficients of relation of 13 , for energies of 6 and $18 \mathrm{MV}$, depth of maximum dose was obtained according to Table 3 .

\section{Discussion}

According to Table.1, by increasing the field size, the percentage of surface dose increases that this could be caused by an increase of the amount of scattering in the larger fields and on the other hand with increased energy, the percentage of surface dose will be reduced due to the reduced backscattered. According to the present findings, amount of kerma on the surface of the skin, as usual, will be increased due to the increase of the field, and will be reduced due to increased energy.

All depths reported in this study, including $\mathrm{d}_{\max }$ have the accuracy within the range of $\pm 3 \mathrm{~mm}$ (the radius of ionization chamber) and the shifting parameter $\mathrm{u}$ assured the accuracy of fitting process and normalization of PDD quantity for all fields to a unique $\mathrm{d}_{\max }$ in each energy, as seen in Table 3 , in low energies, changes of $d_{\text {max }}$ with field size is not very noticeable.

Using Kodak X-Omat radiographic films with an effective depth of measurement of $0.38 \mathrm{~mm}$, Butson obtained surface percentage dose in the energy $6 \mathrm{MV}$ of Varian accelerator $2100 \mathrm{C}$ of fields of $10 \times 10$ up to $30 \times 30 \mathrm{~cm}^{2}$ after extrapolation at depth of zero in fields from $15 \%$ to $38 \%$ (Butson et al., 2004). But in this study using the Eq.(15), PDD value was obtained at the depth of zero in the same fields $30.34 \%$ to $31.57 \%$ that the percentage of surface dose achieved have been within the range of the results of the study conducted by Butson.

Parsai calculated the surface PDD at 6 and $10 \mathrm{MV}$ linear acceleratorVarian 2300 energies of fields of $5 \times 5 \mathrm{~cm}^{2}$ to $25 \times 25 \mathrm{~cm}^{2}$ with the use of extrapolation chamber, parallel plate chamber, a cylindrical chamber and a Monte Carlo simulation. For the field of $10 \times 10 \mathrm{~cm}^{2}$ energyof 6 MV, PDD surface, in different dosimeter are above 16.04, $16.14,48,34.6 \%$, respectively (Ishmael et al., 2008). But in this research, in-depth zero and same field size in energy $6 \mathrm{MV}$, PDD amount equal to $30.34 \%$.

Using Micro-MOSFET at the surface of a phantom in energy $6 \mathrm{MV}$ Varian 21EX Accelerator and field size of $10 \times 10 \mathrm{~cm}^{2}$, Xiang found that the PDD value is $41.2 \%$, and at a depth of $1 \mathrm{~mm}$ is $42 \%$ (Xiang et al., 2007). But in this study, the PDD value at the surface of phantom was $30.34 \%$ and at depth of $1 \mathrm{~mm}$ was $42.12 \%$.

Limitation of application tools in determining the dose at one point reveals the importance of the above relation by the dimensions of chambers or other tools. In this study, with respect to obtaining a quite analytical relationship based on physical principles, with parameters, surface skin dose can be determined. Similarly, the amount of kerma, which is the source and the generator of dose, can be achieved at each point of the irradiated volume. On the other hand, other parameters such as the buildup region thickness and its variations to the existing variables were obtained from the relationship.

\section{Acknowledgements}

This work is Abdalvand's thesis and was supported by Ahvaz Jundishapur university of medical sciences, Iran (Grant number U-93201).

\section{References}

Apipunyasopon L, Phaisangittisakul N, Srisatit S (2013). Equivalent square formula for determining the surface dose of rectangular field from $6 \mathrm{MV}$ therapeutic photon beam. $J$ Appl Clin Med Physics, 14.

Attix FH 2008. Introduction to radiological physics and radiation dosimetry, John Wiley \& Sons.

Bilge H, Cakir A, Okutan M, et al (2009). Surface dose measurements with GafChromic EBT film for 6 and 18MV photon beams. Physica Med, 25, 101-4.

Butson M, Mathur J, Metcalfe P (1997). Skin dose from radiotherapy $\mathrm{X}$-ray beams: The influence of energy. Australasian Radiol, 41, 148-50.

Butson MJ, Cheung T, Peter K, et al (2004). Surface dose extrapolation measurements with radiographic film. Physics Med Biol, 49, 197.

Charles M, Khan Z (1978). Implementation of the ICRP 
recommendation on skin dose measurement using thermoluminescent dosemeters. Physics Med Biol, 23, 972.

Cora S, Francescon P (1995). Accurate build-up and surface dose measurements of megavolt photon beams from variety of accelerators. Phys Med, 11, 17-22.

Devic S, Seuntjens J, Abdel-Rahman W, et al (2006). Accurate skin dose measurements using radiochromic film in clinical applications. Medical Physics, 33, 1116-24.

Ishmael Parsai E, Shvydka D, Pearson D, et al (2008). Surface and build-up region dose analysis for clinical radiotherapy photon beams. Applied Radiat Isotopes, 66, 1438-42.

Jornet N, Ribas M, Eudaldo T (2000). In vivo dosimetry: intercomparison between p-type based and n-type based diodes for the 16-25 MV energy range. Med Physics, 27, 1287-93.

Klein EE, Esthappan J, Li Z (2003). Surface and buildup dose characteristics for 6,10 , and $18 \mathrm{MV}$ photons from an Elekta Precise linear accelerator. J Applied Clin Med Physics, 4, 1-7.

Lin J-P, Chu T-C, Lin S-Y, et al (2001). Skin dose measurement by using ultra-thin TLDs. Applied Radiat Isotopes, 55, 383-91.

Loevinger R (1981). A formalism for calculation of absorbed dose to a medium from photon and electron beams. Medical physics, $8,1-12$.

Nilsson B, Montelius A (1986). Fluence perturbation in photon beams under nonequilibrium conditions. Med Physics, 13, $191-5$.

Ravikumar M, Ravichandran R (2000). Dose measurements in the build-up region for the photon beams from Clinac- 1800 dual energy medical linear accelerator. Strahlentherapie und Onkologie, 176, 223-8.

Scalchi P, Francescon P, Rajaguru P (2005). Characterization of a new MOSFET detector configuration for in vivo skin dosimetry. Medical physics, 32, 1571-8.

Tannous N, Gagnon W, Almond P (1981). Buildup region and skin-dose measurements for the Therac 6 Linear Accelerator for radiation therapy. Medical Physics, 8, 378-81.

Xiang HF, Song JS, Chin DW, et al (2007). Build-up and surface dose measurements on phantoms using micro-MOSFET in 6 and 10MV x-ray beams and comparisons with Monte Carlo calculations. Medical Physics,34, 1266-73. 